\title{
Plasma Levels of Immunoinflammatory Markers in De Novo Coronary Atherosclerosis and Coronary Restenosis Postangioplasty
}

\author{
Alexandre Schaan de Quadros, Jorge Pinto Ribeiro, Waldomiro Carlos Manfroi, Cristiane Leitão, \\ Karen Ordovás, Letícia Weiss, Nadine Clausell
}

Porto Alegre, RS - Brazil

\begin{abstract}
Objective - To compare circulating plasma levels of immunoinflammatory markers in patients with known de novo coronary artery disease and patients with postangioplasty restenosis.
\end{abstract}

Methods - Using enzymatic immunoabsorbent assay, we measured plasma levels of soluble interleukin-2 receptosr, tumor necrosis factor alpha, and soluble tumor necrosis alpha receptors I and II in 11 patients with restenosis postcoronary angioplasty (restenosis group), in 10 patients with primary atherosclerosis (de novo group) who were referred for coronary angiography because of stable or unstable angina, and in 9 healthy volunteers (control group). Levels of soluble interleukin-2 receptors were significantly higher in the de novo group compared with that in the restenosis and control groups. Levels were also higher in the restenosis group compared with that in the control group. Plasma levels of tumor necrosis alpha and receptor levels were significantly higher in the de novo group compared to with that in the restenosis and control groups, but levels in the restenosis group werenot different from that in the controls.

Conclusion - Coronary artery disease, either primary or secondary to restenosis, is associated with significant immunoinflammatory activity, which can be assessed by examining the extent of circulating plasma levels of inflammatory markers. Moreover, patients with de novo lesions appear to have increased inflammatory activity compared with patients with restenosis.

Key words: coronary artery disease, inflammation, tumor necrosis factor alpha, interleukin-2

Hospital de Clínicas de Porto Alegre - Federal University of Rio Grande do Sul, Porto Alegre, Brazil

Mailing address: Nadine Clausell - Serviço de Cardiologia - Hospital de Clínicas de Porto Alegre - Rua Ramiro Barcelos, 2350 - 90035-003 - Porto Alegre, RS Brazil - E-mail:clausell@portoweb.com.br
Immunoinflammatory mechanisms are thought to be involved in the pathogenesis of restenosis after coronary balloon angioplasty. Mechanical injury to the vascular wall caused by angioplasty stimulates the production of growth factors and cytokines by inflammatory cells attracted from the peripheral blood to the injury site, and by vascular cells that become activated ${ }^{1}$. This "restenosis cascade" involves cytokines, such as tumor necrosis factor alpha, interleukin-1 beta, and interleukin-2, which may contribute to the development of intimal hyperplasia and vascular remodeling ${ }^{2}$. In previous studies, we demonstrated that tumor necrosis alpha expression was increased in coronary restenotic lesions retrieved by atherectomy compared with expression in de novo lesions ${ }^{3}$. In addition, Pietersma et al. ${ }^{4}$ demonstrated that increased production of interleukin- 1 beta by circulating monocytes predicted restenosis.

Although these studies strongly suggest that immunoinflammatory mechanisms can be pivotal in the development of restenosis, it remains unclear whether intraplaque events, such as increased cytokine content, are mirrored by increased circulating levels of these biological markers in patients presenting with coronary restenosis. Therefore, in this study we measured levels of tumor necrosis alpha and its soluble receptors I and II, and soluble interleukin-2 receptors in the peripheral blood of patients with coronary restenosis postangioplasty and compared that with levels in patients with de novo coronary lesions and in normal individuals.

\section{Methods}

We analyzed eleven patients with coronary restenotic lesions, 10 patients with de novo coronary lesions and 9 normal individuals who served as controls. Patients were enrolled in the study as they were referred to the catheterization laboratory for coronary angiography by their attending cardiologists. All patients were interviewed and clinical pre- 
sentation was classified as stable or unstable angina class IB, IIB, or III B according to Braunwald's criteria ${ }^{5}$. Use of cardiac drugs as prescribed by the attending cardiologists was recorded. Exclusion criteria were: systemic inflammatory disease, heart failure, ejection fraction less than $40 \%$, decompensated diabetes mellitus, current use of antiinflammatory (except for aspirin) or immunosupressive drugs, acute myocardial infarction or surgery in the last three months, systemic infections, or neoplasia. To minimize chances of asymptomatic coronary artery disease, the control group was formed by subjects under 35 years of age, with no symptoms or signs of cardiac disease, absence of risk factors for coronary artery disease, and no significant medical history. They did not undergo coronary angiography. The study protocol was previously approved by the Ethics and Research Committee of Hospital de Clínicas de Porto Alegre, and we obtained written informed consent from all subjects prior to enrollment.

Clinicians used Judkin's technique to perform coronary arteriography on the patients. The presence and severity of coronary angiographic lesions were evaluated from at least three projections by one investigator who was blinded to the clinical characteristics of the patients. Coronary restenosis was defined as the recurrence of a $50 \%$ stenosis in the site of a previously dilated vessel. Patients in the group of de novo coronary atherosclerosis had a $70 \%$ stenosis in at least one coronary artery. The number of affected vessels was also recorded.

Patients had $20 \mathrm{~mL}$ of blood drawn from a venous site to an EDTA-containing tube immediately prior to coronary angiography. Control subjects had blood drawn in a quiet laboratory room. Samples were immediately centrifuged at 2000 rpm for 10 minutes, and the plasma was stored at $-20^{\circ} \mathrm{C}$ for up to 6 months for future combined analysis. Enzyme-linked immunoabsorbent assays were then run using duplicate samples, to minimize interassay variability, to measure plasma circulating levels of soluble interleukin-2 receptors (sensitivity $<6 \mathrm{pg} / \mathrm{mL}$; range $78.1-5000 \mathrm{pg} / \mathrm{mL}$ ), tumor necrosis alpha (sensitivity $<4.4 \mathrm{pg} / \mathrm{mL}$; range $15.6-1000 \mathrm{pg} / \mathrm{mL}$ ), soluble receptor I of tumor necrosis alpha (sensitivity $<3 \mathrm{pg} / \mathrm{mL}$; range $7.8-$ $500 \mathrm{pg} / \mathrm{mL}$ ), soluble receptor II of tumor necrosis alpha (sensitivity $<1 \mathrm{pg} / \mathrm{mL}$; range $7.8-500 \mathrm{pg} / \mathrm{mL}$ ) (R\&D Systems, Minneapolis). Lipid profile, creatine phosphokinase (CPK), and MB fraction were also measured in all subjects.

Continuous variables are expressed as means \pm SD. Tumor necrosis alpha was not normally distributed and is presented as median [range]. Differences among the three groups were analyzed by ANOVA with Scheffé's procedure, except for tumor necrosis alpha, for which the KruskallWallis test was used. Associations among variables were evaluated using Pearson's correlation coefficient or Spearman's rank-order correlation coefficient, and differences between groups of patients were analyzed by the Students $t$ test or Fisher's exact test for categoric variables. A p value of $<0.05$ was considered significant for all tests. Linear regression models were used to analyze variables adjusting for baseline clinical differences among groups.

\section{Results}

Characteristics of patients and control subjects are shown in the Table I. According to inclusion criteria, normal individuals were significantly younger than both groups of patients. Unstable angina accounted for the totality of cases in the de novo lesions group, but only $7 / 11$ patients in the restenosis group had unstable angina; however, this was not statistically significant. The time from the last episode of angina in the group of patients with restenosis was significantly shorter than that of patients with de novo lesions $(77 \pm 214$ versus $124 \pm 144$ hours; $p<0.05)$. No statistically significant differences occurred regarding the use of cardiovascular drugs, which included beta-blockers, aspirin, nitrates, calcium channel blockers, ticlopidin, or intravenous heparin. The lipid profile was also similar between the two groups of patients, and no change in cardiac enzyme profiles were observed. The mean elapsed time from coronary angioplasty in the group of patients with restenosis was $165 \pm 35$ days.

Significantly more patients in the de novo group presented with one vessel disease compared with the restenosis group (Table I). Other angiographic characteristics, such as proximal left anterior descending artery involvement and severity of stenosis, were not significantly different between the groups.

Individual data as well as mean values for immunoinflamatory markers are presented in the Figure 1. Levels of soluble interleukin-2 receptors were significantly higher in the group of patients with de novo coronary lesions compared with that in normal controls and patients with restenosis $(2283 \pm 542 \mathrm{pg} / \mathrm{mL}, 1640 \pm 576 \mathrm{pg} / \mathrm{mL}$ and $796 \pm 470 \mathrm{pg} / \mathrm{mL}$, respectively, $\mathrm{p}<0.05)$. In addition, soluble interleukin-2 receptor levels were also higher in the restenosis group compared with levels in the normal controls. Plasma levels of tumor necrosis alpha were significantly higher in patients with de novo lesions $[0.65 \mathrm{pg} / \mathrm{mL}(0-3.0 \mathrm{pg} / \mathrm{mL})]$ compared with that in the restenosis group $[0 \mathrm{pg} / \mathrm{mL}(0-2.8 \mathrm{pg} / \mathrm{mL}) ; \mathrm{p}<0.05]$ and compared with that in the normal controls $[0 \mathrm{pg} / \mathrm{ml}(0-0.3 \mathrm{pg} /$ $\mathrm{mL}) ; \mathrm{p}<0.05]$. Circulating levels of soluble tumor necrosis alpha receptor I were also significantly higher in the group of patients with de novo lesions compared with that in patients with restenosis $(1223 \pm 194 \mathrm{pg} / \mathrm{mL}$ versus $1021 \pm 108$ $\mathrm{pg} / \mathrm{mL} ; \mathrm{p}<0.05)$ and compared with that in normal controls $(888 \pm 162 \mathrm{pg} / \mathrm{mL} ; \mathrm{p}<0.05)$. As for circulating levels of soluble tumor necrosis alpha receptor II, values were significantly higher in the group of patients with de novo lesions compared with that in patients with restenosis $(2958 \pm 716 \mathrm{pg} / \mathrm{mL}$ versus $2267 \pm 447 \mathrm{pg} / \mathrm{mL}$; $\mathrm{p}<0.05$ ) and compared with that in normal controls $(2123 \pm 345 \mathrm{pg} / \mathrm{mL} ; \mathrm{p}<0.05)$. Taken together, these results demonstrate increased levels of these inflammatory markers in the group of patients with de novo lesions compared with that in both other groups. In addition, levels of these markers observed in patients with restenosis were not statistically different compared with levels in the normal controls, except for soluble interleukin-2 receptor levels. Significant correlations between tumor necrosis alpha and soluble tumor necrosis alpha receptor $\mathrm{I}(\mathrm{r}=0.6 ; \mathrm{p}<0.05)$ and 
between tumor necrosis alpha and soluble tumor necrosis alpha receptor II ( $\mathrm{r}=0.6 ; \mathrm{p}<0.05)$ were observed. Similarly, soluble receptor levels correlated with each other $(r=0.85$; $\mathrm{p}<0.05)$. After adjustment for differences in age among groups, results remained unchanged.

\section{Discussion}

Atherosclerosis can be considered an immunoinflammatory process evolving over a number of years according to the presence or absence of risk factors and genetic background ${ }^{6}$. Restenosis can be viewed as an accelerated form of this process with associated wound-healing characteristics ${ }^{2}$. In this study, we demonstrated an enhanced state of inflammatory activity detected in the plasma of patients presenting with coronary artery disease syndromes secondary to both de novo or restenotic lesions compared with that in the normal controls.

Patients enrolled in this study constituted a typical group of individuals referred by their attending cardiologists for coronary angiography for known or suspected coronary artery disease. No major clinical or laboratory differences were observed between the two groups of patients, except for an increased number of patients presenting with unstable angina in the group with de novo lesions. Although this difference did not reach statistical significance, this is in keeping with the notion that, overall, restenotic lesions are less likely to cause unstable syndromes ${ }^{7}$. Use of medications was also similar in both groups at the time of blood sampling, except for two patients receiving heparin intravenously in the de novo atherosclerosis group.

Atherogenesis can be viewed as an inflammatory process in which vascular cells can play important roles mediating several immunoinflammatory mechanisms secondary to endothelial injury. Endothelial cells express adhesion molecules in their surface that will induce T cells and monocytes/macrophages to adhere to the vascular endothelial surface and subsequently migrate to the subendothelial space. Increased plasma levels of different inflammatory media-

\begin{tabular}{|c|c|c|c|}
\hline & De Novo & Restenosis & Controls \\
\hline $\mathrm{N}$ & 10 & 11 & 9 \\
\hline Age (years) & $64 \pm 6$ & $58 \pm 13$ & $28 \pm 4 * *$ \\
\hline Gender (male) & 6 & 7 & 10 \\
\hline Unstable angina $(\mathrm{n})$ & 10 & 7 & \\
\hline $\begin{array}{l}\text { Previous acute myocardial } \\
\text { infarction }(n)\end{array}$ & 1 & 5 & \\
\hline LDL-Cholesterol (mg/dl) & $123 \pm 28$ & $141 \pm 47$ & $121 \pm 39$ \\
\hline HDL-Cholesterol (mg/dl) & $41 \pm 9$ & $38 \pm 9$ & $42 \pm 9$ \\
\hline Severity of stenosis $(\%)$ & $97 \pm 4$ & $88 \pm 14$ & \\
\hline One vessel disease (n) & 9 & $2 *$ & \\
\hline $\begin{array}{l}\text { Proximal LAD } \\
\text { involvement }(\mathrm{n})\end{array}$ & 5 & 3 & \\
\hline
\end{tabular}

tors, indicative of this inflammatory process, have been identified in established coronary artery disease ${ }^{8-11}$. Our group has also recently demonstrated that patients with stable and unstable angina with significant coronary artery disease by angiography and even patients without flow-limiting coronary stenosis but presenting with chest pain may have increased plasma levels of adhesion molecules ${ }^{12}$. In fact, Ridker and co-workers ${ }^{13}$ have recently shown that increased levels of intracellular adhesion molecule- 1 in healthy men are predictors of future acute myocardial infarction.

In the present study, yet investigating different markers of inflammatory activity, we also showed that patients presenting with stable or unstable angina feature a pattern consistent with an enhanced state of immunoinflammatory activation. In our study, the source of increased levels of the different markers we examined is unclear. Because both vascular and more likely T cells and monocytes present in the atherosclerotic plaque can synthesize tumor necrosis alpha, it can be speculated that this cytokine could be initially formed in the plaque and subsequently released in the circulation. In fact, Rus et al. ${ }^{14}$ have recently shown increased $C$ protein and interleukin-6 levels eluted from human arterial wall with atherosclerotic disease. On the other hand, Liuzzo et al. ${ }^{15}$ have indicated that an enhanced immunoinflammatory response in unstable angina patients could be secondary to nonspecific stimuli. Similarly, soluble interleukin-2 receptors, which are derived from activated T cells, could also be produced by intraplaque lymphocytes or by activated circulating T cells. We have previously shown that, compared with primary lesions, restenotic coronary lesions subsequent to both atherectomy or balloon angioplasty feature increased expression of tumor necrosis alpha ${ }^{3}$. This was associated with a higher number of T cells in these lesions. Others have also suggested that an enhanced state of inflammatory activity could be important to the development of restenosis ${ }^{4,16,17}$. Whether these features can be translated into a clinical situation of patients presenting with coronary syndromes and detected in circulating plasma of patients remains unclear. Our study aimed to characterize patients with known restenotic lesions or de novo lesions regarding circulatory levels of different markers of inflammation. The pattern of tumor necrosis alpha and its soluble receptors and that of soluble interleukin- 2 receptors consistently indicated an enhanced inflammatory activity in the group of patients with de novo lesions, when compared with that in controls or in patients with restenosis. This is in contrast to our previous immunohistochemical studies on both primary and restenotic plaques. It is possible that differences in the expression of tumor necrosis alpha, which has a known short half-life ${ }^{18,19}$, observed in atherectomy specimens do not have the magnitude to be maintained when clinical studies measuring this peptide in the circulating plasma are carried out. In addition, all of the patients in the de novo group from the present study had unstable angina compared with $7 / 11$ in the restenosis group. Although this difference was not statistically significant, the known ruptured/fissured plaque associated with unstable angina may have contributed to a more pronounced inflammatory 


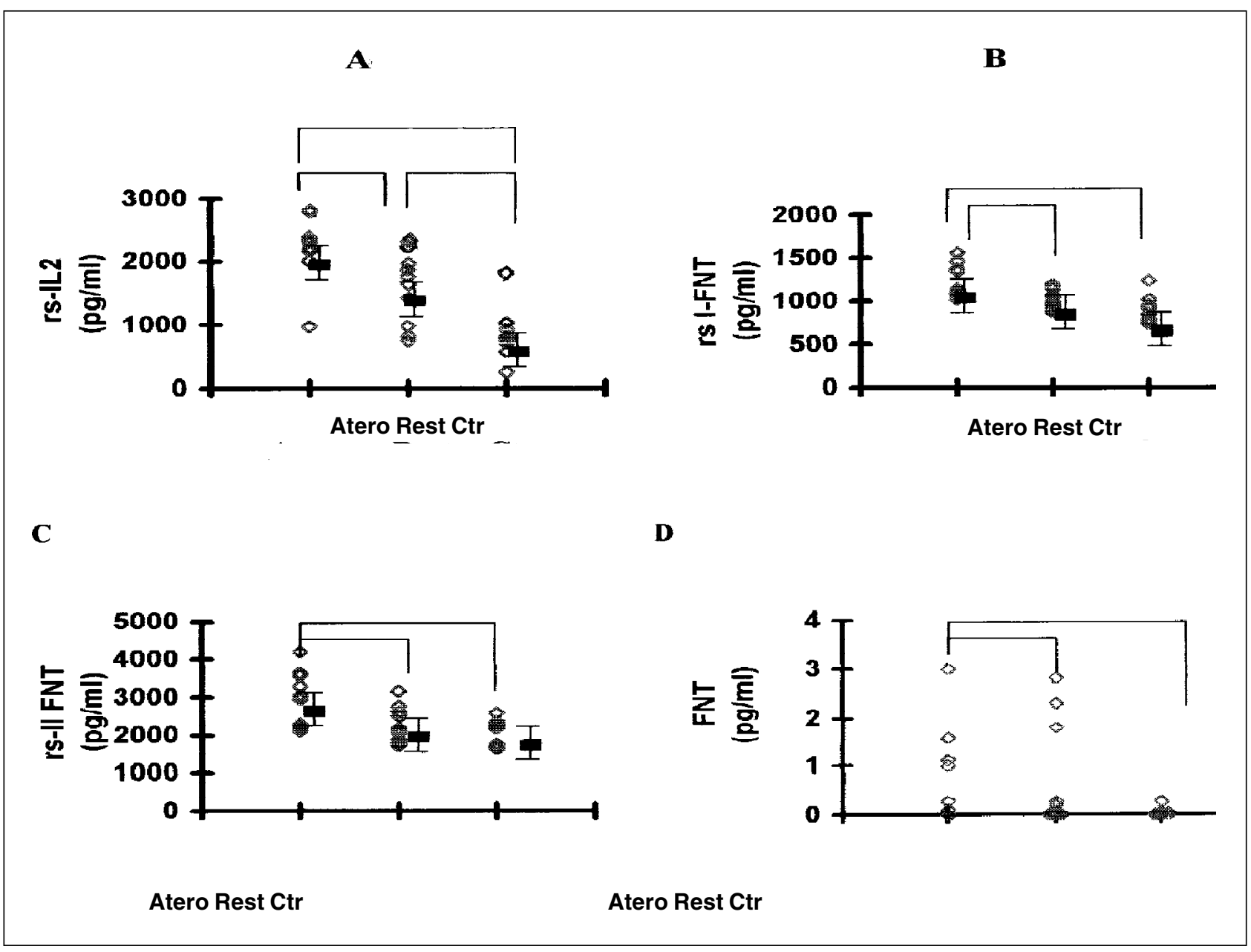

Fig. 1 - Individual data for immunoinflammatory markers in patients with de novo coronary lesions (de novo), patients with restenosis (Rest), and controls (Ctr). Groups connected by lines are significantly different. A) Plasma levels of soluble receptor of interleukin-2 (sr-IL2); B) plasma levels of soluble receptor I of tumor necrosis factor alpha (sr I-TNF-a); C) plasma levels of soluble receptor II of tumor necrosis alpha (sr II-TNFa); D) plasma levels of tumor necrosis alpha (TNFa). Data and mean \pm SD (except for TNF-a).

reaction in this group of patients, offsetting a potential increased inflammatory activity expected to be present in patients with restenotic lesions.

Our data should be interpreted in the light of specific study limitations. First, our limited sample size may not support definitive answers, but regardless of the number of study individuals, our data collectively show a consistent pattern of heightened inflammatory activity in the de novo group as opposed to the restenosis group. Second, we chose a control group that was significantly younger than both other groups. While this may have influenced our analysis, interleukin 2 soluble receptor levels may correlate with age only up to 4 years when the immune system is still being developed ${ }^{20}$. Tumor necrosis factor alpha was not influenced by age in similar studies ${ }^{21,22}$. Third, as mentioned above, our data may not reflect intraplaque events, because only peripheral blood was studied. It can be speculated that blood sampling from the coronary sinus could more appropriately identify differences occurring inside the atherosclerotic plaque. Finally, because coronary syndromes can be complex, it would be interesting to investigate patients presenting with clinical syndromes as comparable as possible (all stable angina or all unstable angina patients with primary and restenotic lesions).

In conclusion, our study reinforces the notion that coronary artery disease is associated with a pattern of immunoinflammatory activity and suggests that this can be assessed by measuring plasma levels of tumor necrosis alpha and its receptors and soluble interleukin- 2 receptors. Our data also indicate significant increases in circulatory levels of these markers in patients with primary atherosclerosis when compared with that in patients with restenotic lesions or in normal controls. Finally, a moderate degree of immunoinflammatory activity was shown in patients with restenosis as increased levels of soluble interleukin-2 receptors only were observed in this group compared with controls.

\section{Acknowledgements}

We are thankful to Dr. Karen Prado and Dr. Jarbas Oliveira for their support to carry out the immunoabsorbent assays. We are also grateful to Dr. Murilo Foppa for his suggestions and help in the initial phases of the study. Statistical support was provided by Ms. Luciana Bertoldi Nucci. 


\section{Referências}

1. Serrano CV, Ramires JA, Venturinelli M, et al. Coronary angioplasty results in leukocyte and platelet activation with adhesion molecule expression. Evidence of inflammatory responses in coronary angioplasty. J Am Coll Cardiol 1997; 29: 1276-83.

2. Libby P, Schwartz D, Brogi E, Tanaka H, Clinton SK. A cascade model for restenosis. A special case of atherosclerosis progression. Circulation 1992; 86(suppl 3): 47-52.

3. Kelso A. Cytokines: structure, function and synthesis. Curr opin immunol 1989; $2: 215-25$.

4. Le J, Vilcek J. Tumor necrosis factor and interleukin-1: cytokines with multiple overlapping biological activities. Lab Invest 1987; 56: 234-48.

5. Clausell N, Molossi S, Sett S, Rabinovitch M. In vivo blockade of tumor necrosis factor-alpha in cholesterol-fed rabbits after cardiac transplant inhibits acute coronary artery neointimal formation. Circulation 1994; 89: 2768-79.

6. Dinarello CA, Mier JW. Lymphokines. N Engl J Med 1987; 317: 940-5.

7. Rubin LA, Nelson DL. The soluble interleukin-2 receptor: biology, function, and clinical application. Ann Intern Med 1990; 113: 619-27.

8. Clausell N, de Lima VC, Molossi S, et al. Expression of tumour necrosis factor alpha and accumulation of fibronectin in coronary artery restenotic lesions retrieved by atherectomy. Br Heart J 1995; 73: 534-9.

9. Pietersma A, Kofflard M, de Wit LE, et al. Late lumen loss after coronary angioplasty is associated with the activation status of circulating phagocytes before treatment. Circulation 1995; 91: 1320-5.

10. Braunwald E. Unstable angina: a classification. Circulation 1989; 80: 410-14

11. Ross R. Mechanism of disease: atherosclerosis - an inflammatory disease. $\mathrm{N}$ Engl J Med 1999; 340: 115-26.

12. Nobuyoshi M, Kimura T, Oshishi H, et al. Restenosis after percutanous transluminal coronary angioplasty: pathological observations in 20 patients. J Am Coll Cardiol 1991; 17: 433-9.

13. Blum A, Sclarovsky S, Shohat B. T lymphocyte activation in stable angina pectoris and after percutaneous transluminal coronary angioplasty. Circulation 1995; 91: 20-2.

14. Schumacher M, Halwachs G, Tatzber F, et al. Increased neopterin in patients with chronic and acute coronary syndromes. J Am Coll Cardiol 1997; 30: 703-7.
15. Hasdai D, Scheinowitz M, Leibovitz E, Sclarovsky S, Eldar M, Barak V. Increased serum concentrations of interleukin- $1 \mathrm{~b}$ in patients with coronary artery disease. Heart 1996; 76: 24-8.

16. Neri Serneri GG, Prisco D, Martini F, et al. Acute T-cell activation is detectable in unstable angina. Circulation 1997; 95: 1806-12.

17. Clausell N, Prado K, Ribeiro JP. Increased plasma levels of soluble vascular cellular adhesion molecule- 1 in patients with chest pain and angiographically normal coronary arteries. Int J Cardiol 1999; 68: 275-80.

18. Ridker PM, Hennekens CH, Roitman Johnson B, Stampfer MJ, Allen J. Plasma concentration of soluble intercellular adhesion molecule 1 and risks of future myocardial infarction in apparently healthy men. Lancet 1998; 351: 88-92.

19. Rus H, Niculescu F. Inflammatory response in unstable angina Circulation 1999 100: 98

20. Liuzzo G, Buffon A, Biasucci LM, et al. Enhance inflammatory response to coronary angioplasty in patients with severe unstable angina. Circulation 1998; 98 : 2370-6.

21. Moreno PR, Bernardi VH, Lopez Cuellar J, et al. Macrophage infiltration predicts restenosis after coronary intervention in patients with unstable angina. Circulation 1996; 94: 3098-102.

22. Lima VC, Gottlieb AI, Clausell N, et al. Analysis of atherosclerotic plaques obtained by coronary atherectomy: foam cells correlated positively with subsequent restenosis. Cardiovasc Pathol 1996; 5: 265-9.

23. Packer M. Is tumor necrosis factor an important neurohormonal mechanism in chronic heart failure? Circulation 1995; 92: 1379-82.

24. Mueller AR, Platz K, Haak M, et al. The release of cytokines, adhesion molecules, and extracellular matrix parameters during and after reperfusion in human liver transplantation. Transplantation 1996; 62: 1118-26.

25. Rubin LA, Nelson DL. The soluble interleukin-2 receptor: biology, function, and clinical application. Ann Intern Med 1990; 113: 619-27.

26. Mendall MA, Patel P, Asante M, et al. Relation of serum cytokine concentrations to cardiovascular risk factors and coronary heart disease. Heart 1997; 78: 273-7.

27. Roubenoff R, Harris TB, Abad LW, et al. Monocyte cytokine production in an elderly population: effect of age and inflammation. J Gerontol A Biol Sci Med Sci.1998; 53: M20-6. 